\title{
FORMULAÇÃO DE LEITE EM PÓ ENRIQUECIDO COM FERRO
}

FABRÍCIAQUEIROZ MENDES* JOSÉ CARLOS GOMES**

THIAGO SOUZA BARBOSA ${ }^{\star \star *}$

MARCO ANTÔNIO SARTORI*

MARIA GORETI DEALMEIDA OLIVEIRA***

\begin{abstract}
Este trabalho objetivou estudar o enriquecimento de leite em pó com duas fontes diferentes de ferro: sulfato ferroso e ferro aminoácido quelato. As fontes de ferro foram adicionadas antes e após a secagem do leite. Foram estudadas a reconstituição, a estabilidade oxidativa e a biodisponibilidade das fontes de ferro. Quanto à forma de enriquecimento não houve diferença entre as características estudadas. Ambos os processos tecnológicos mostraram-se simples e de fácil execução em laboratório. A estabilidade oxidativa do leite enriquecido foi maior quando se utilizou ferro aminoácido quelato e ambas as fontes diferiram do controle. O ferro aminoácido quelato apresentou maior biodisponibilidade in vitro $(31,81 \%)$ que o sulfato ferroso $(17,41 \%)$. A biodisponibilidade in vivo, deduzida, foi de $15,45 \%$ para o ferro aminoácido quelato e de $8,66 \%$ para o sulfato ferroso. O ferro aminoácido quelato mostrou melhor estabilidade oxidativa e melhor biodisponibilidade comparado com o sulfato ferroso, constituindo boa fonte para o enriquecimento do leite em pó.
\end{abstract}

PALAVRAS-CHAVE: ANEMIA FERROPRIVA; DISPONIBILIDADE NUTRICIONAL; OXIDAÇÃO QUÍMICA; LEITE EM PÓ.

\footnotetext{
* Mestre em Ciência e Tecnologia de Alimentos, Universidade Federal de Viçosa (UFV), Visoça, MG (email: fabriciaqm@yahoo.com.br; marcosartori10@yahoo.com.br).

** Bacharel em Química, Doutor em Análise Química de Alimentos, Professor, UFV, Visoça, MG (email:jcgomes@ufv.br).

*** Graduando em Engenharia de Alimentos, UFV, Visoça, MG (e-mail:barbosaufv@yahoo.com.br).

*** Licenciada e Bacharel em Química, Doutora em Bioquímica e Imunologia, Pós-Doutora em Enzimologia, Professora, UFV, Visoça, MG (e-mail:malmeida@ufv.br).
} 


\section{INTRODUÇÃO}

A anemia ferropriva, decorrente da deficiência alimentar de ferro, é a mais comum das carências nutricionais e atinge quase dois bilhões de pessoas em todo o mundo, correspondendo a aproximadamente $36 \%$ da população mundial (LIMA et al., 2004). As crianças, nos primeiros anos de vida, e as gestantes constituem os grupos mais vulneráveis à anemia (BUONGERMINO SOUZA, CORNBLUTH SZARFAC e PACHECO SOUZA, 1997).

As consequências da anemia incluem alterações na capacidade de trabalho físico, prejuízos no desenvolvimento mental e psicomotor das crianças, aumento da mortalidade materna e infantil, baixo peso da criança ao nascer, parto prematuro e redução da resistência às infecções (OLIVARES e WALTER, 2003; TUMA et al., 2003).

Os estoques de ferro no organismo são controlados pela absorção, que deve compensar a perda diária de ferro. Em homens adultos as perdas de ferro são de aproximadamente 1,0 mg por dia devido a sangramentos, descamação de células intestinais e epiteliais, perdas pela bile e urina. Nas mulheres em período fértil, há perdas adicionais de $0,5 \mathrm{mg}$ de ferro por dia devido à menstruação (MACPHAIL, 2001).

Considerando a perda diária e que a média de absorção de ferro presente nas dietas é de 10\%, a ingestão diária recomendada (IDR) para crianças de 0-5 meses é de $6 \mathrm{mg}$ por dia e de seis meses a dez anos de $10 \mathrm{mg}$ por dia. Para homens entre 11 e 18 anos, a IDR é de $12 \mathrm{mg}$ por dia e para maiores de 19 anos de $10 \mathrm{mg}$ por dia. Para mulheres entre 11 e 50 anos, ou lactação, a recomendação é de $15 \mathrm{mg}$ por dia, maiores de 51 anos de $10 \mathrm{mg}$ por dia e para gestantes de $30 \mathrm{mg}$ por dia (NRC, 1989).

A fortificação de alimentos tem sido considerada como a melhor forma de prevenir a deficiência de ferro, quando a dieta não fornece a quantidade necessária desse mineral. Entretanto, alguns fatores devem ser levados em consideração antes da fortificação de algum alimento com ferro como a biodisponibilidade da forma do ferro escolhida e o consumo do alimento pela população alvo. Além disso não podem ocorrer alterações no alimento como a modificação da cor, desenvolvimento de sabor estranho e sedimentação de compostos pouco solúveis (SOGLIA, 1996).

O sulfato ferroso constitui a substância mais utilizada para tratamento da anemia devido ao baixo custo e elevada biodisponibilidade. Apresenta cor esverdeada, sabor metálico e odor irritante. Em doses elevadas pode causar problemas de intoxicação (SAKAMOTO, 2003). O ferro aminoácido quelato é um composto de ferro ligado a duas moléculas de glicina, resultando em duplo anel heterocíclico. O grupo $\alpha$-carboxílico da glicina é ligado ao ferro mediante ligação iônica, enquanto que o grupo $\alpha$-amino é ligado ao metal por ligação covalente (OLIVARES et al., 1997).

Os compostos quelatos de ferro, como o ferro aminoácido quelato (biglicinato de ferro), NaFeEDTA, ferro maltose e ferroprotinato apresentam a mesma biodisponibilidade do sulfato ferroso, porém provocam menores alterações no alimento. Os quelatos devem se ligar ao ferro de forma a permitir sua transferência para outros ligantes presentes na membrana ou citosol do enterócito, mas evitando sua liberação em sítios que o ferro possa agir formando radicais livres (SAKAMOTO, 2003). Além disso, a solubilidade desses quelatos deve ser alta nas proximidades dos sítios de absorção (CLAUD e FREITAS, 1994).

O objetivo do presente trabalho foi desenvolver leite em pó enriquecido com ferro, determinar o efeito da adição de ferro na oxidação de gordura do produto desenvolvido e determinar a biodisponibilidade do leite em pó enriquecido com ferro.

\section{MATERIAL E MÉTODOS}

O preparo das amostras, as análises físico-químicas dos produtos e o teste de biodisponibilidade in vitro foram conduzidos no Laboratório de Análise de Alimentos, Departamento de Tecnologia de Alimentos, Universidade Federal de Viçosa (Minas Gerais). 


\subsection{PREPARO DAS AMOSTRAS}

As fontes de ferro adicionadas ao leite foram ferro aminoácido quelato (Albion Laboratories, Inc. Clearfield-Utah-USA) e sulfato ferroso heptahidratado, $\mathrm{FeSO}_{4} \cdot 7 \mathrm{H}_{2} \mathrm{O}$ (Vetec Com. \& Repr. Rio de Janeiro-BR), ambos contendo $20 \%$ de ferro.

As duas fontes de ferro foram adicionadas ao leite antes e após a secagem, sendo que leite em pó sem adição de ferro foi tomado como referência. Os cinco tratamentos foram preparados em três repetições.

Para os tratamentos fortificados após a secagem usou-se leite em pó comercial, sendo adicionados $12 \mathrm{mg}$ de ferro para $100 \mathrm{~g}$ de amostra. Desta forma, após reconstituição ( $26 \mathrm{~g}$ ou duas colheres de sopa de leite em pó para $200 \mathrm{~mL}$ de água) cada $100 \mathrm{~mL}$ do produto pronto para consumo fornecerá $1,5 \mathrm{mg}$ de ferro (equivalente a $15 \%$ da IDR para crianças). Assim, o produto pode ser considerado enriquecido ou fortificado de acordo com a Portaria $n^{\circ} 31$, de 13 de janeiro de 1998, da Agência Nacional de Vigilância Sanitária, (BRASIL, 1998).

Para os tratamentos fortificados antes da secagem, 1,5 mg de ferro foram adicionados a cada $100 \mathrm{~mL}$ de leite tipo C pasteurizado proveniente do Laticínio-Escola da Universidade Federal de Viçosa. As amostras foram concentradas até redução de $50 \%$ do volume e desidratadas em Spray-dryer (Mini Spray-dryer, Büchi Switzerland, B-191), com temperatura de entrada do ar em torno de $180^{\circ} \mathrm{C} \pm 1^{\circ} \mathrm{Ce}$ temperatura de saída em torno de a $80^{\circ} \mathrm{C} \pm 1^{\circ} \mathrm{C}$.

A Tabela 1 mostra as fontes de ferro e a forma de secagem de cada tratamento.

\section{TABELA 1 - RESUMO DOS TRATAMENTOS APLICADOS}

\begin{tabular}{lcc}
\hline Tratamentos & Fonte de ferro & Forma de secagem \\
\cline { 2 - 3 } A (Controle) & & FeSO \\
B & $\mathrm{FeSO}_{4}$ & Após a adição da fonte de ferro \\
C & Antes da adição da fonte de ferro \\
D & Ferro aminoácido quelato & Após a adição da fonte de ferro \\
E & Ferro aminoácido quelato & Antes da adição da fonte de ferro \\
\hline
\end{tabular}

\subsection{TEOR DE FERRO}

As concentrações de ferro no leite em pó foram determinadas após mineralização de $1 \mathrm{~g}$ de amostra por via úmida, utilizando-se mistura digestora nitro-perclórica na proporção de 3:1. Aqueceu-se a solução em chapa até fervura branda, mantendo-se essa condição até a formação de solução límpida. Após digestão, a solução foi transferida para balão de $100 \mathrm{~mL}$ e o volume completado com água destilada. O teor de ferro foi determinado por colorimetria no comprimento de onda de $510 \mathrm{~nm}$ do complexo formado pelo íon $\mathrm{Fe}^{+2}$ com ortofenantrolina, conforme descrito por GOMES et al. (2003). Empregou-se espectrofotômetro SHIMADZU, modelo UV mini 1240 e utilizou-se curva padrão previamente estabelecida.

\subsection{EFEITO NA OXIDAÇÃO DE GORDURA}

A análise dos produtos secundários de oxidação de gordura foi realizada pelo índice de ácido tiobarbitúrico (TBA), conforme descrito nas Normas Analíticas do Instituto Adolfo Lutz (IAL, 1985). As amostras foram dissolvidas em clorofórmio e $5 \mathrm{~mL}$ dessa solução retirados e colocados em tubo de centrífuga com $5 \mathrm{~mL}$ de ácido tricloroacético a 10\%. A mistura foi centrifugada (International Centrifuge, modelo K, International equipament Co.) a 5000 x g por vinte minutos. Adicionou-se ácido tiobarbitúrico 
ao sobrenadante que foi colocado em água fervente por 10 minutos. Resfriou-se a solução e procedeuse a leitura no comprimento de onda de $530 \mathrm{~nm}$ (em espectrofotômetro SHIMADZU, modelo UV mini 1240), usando água destilada como referência.

\subsection{BIODISPONIBILIDADE IN VITRO}

Estimou-se a biodisponibilidade in vitro do ferro no leite em pó pelo método descrito por RAO e PRABHAVATHI (1978). As amostras foram incubadas a $37^{\circ} \mathrm{C}$ por 90 minutos em solução de pepsina$\mathrm{HCl}(0,5 \%$ de pepsina em $\mathrm{HCl} \mathrm{0,1} \mathrm{N),} \mathrm{pH} \mathrm{1,35.} \mathrm{Após} \mathrm{a} \mathrm{incubação,} \mathrm{o} \mathrm{pH} \mathrm{foi} \mathrm{ajustado} \mathrm{para} \mathrm{7,5} \mathrm{com}$ $\mathrm{NaOH}$ e as amostras centrifugadas (International Centrifuge, modelo K, International equipament $\mathrm{Co}$.) a 5000 x g por 30 minutos. As amostras foram filtradas e o teor de ferro ionizado determinado por reação colorimétrica com ortofenantrolina, conforme descrito por GOMES et al. (2003). Calculo-se a percentagem de ferro ionizável em pH 7,5, ou ferro biodisponível, em relação aos teores de ferro determinados experimentalmente para as amostras. Após determinada a biodisponibilidade in vitro, a biodisponibilidade in vivo foi deduzida a partir da equação de RAO e PRABHAVATHI (1978):

$$
V=0,4717 \mathrm{~T}+0,450^{\circ}
$$

Na qual: $V$ = Biodisponibilidade in vivo;

$\mathrm{T}=$ Biodisponibilidade in vitro.

\subsection{ANÁLISES ESTATÍSTICAS}

Empregou-se o delineamento experimental inteiramente casualisado, com três repetições por tratamento. As análises estatísticas foram processadas com auxílio dos softwares Saeg 9.1 (EUCLYDES, 1983). Os resultados foram submetidos à análise de variância, com teste $F$, realizandose o teste de Tukey para as amostras que obtiveram significância no teste $\mathrm{F}$ ao nível 1\%.

\section{RESULTADOS E DISCUSSÃO}

Todos os tratamentos apresentaram teor de ferro superior a 15\% da IDR para $100 \mathrm{~mL}$ de produto pronto para consumo, preparado na proporção de $26 \mathrm{~g}$ de leite em pó para $200 \mathrm{~mL}$ de leite reconstituído (Tabela 2). De acordo com a Portaria ${ }^{\circ} 31$ da Agência Nacional de Vigilância Sanitária (BRASIL, 1998), o leite em pó desenvolvido pode ser considerado enriquecido ou fortificado com ferro.

\section{TABELA 2 - TEOR DE FERRO DETERMINADO POR ESPECTROFOTOMETRIA APÓS REAÇÃO COM ORTOFENANTROLINA}

\begin{tabular}{lcc} 
Tratamentos & Teor de ferro determinado $(\mathrm{mg} / \mathrm{g})$ & $\begin{array}{c}\text { \% IDR de ferro por } 100 \mathrm{~mL} \text { de } \\
\text { produto pronto para consumo }\end{array}$ \\
\hline A & $0,026^{\mathrm{a}} \pm 0,009$ & 3,25 \\
B & $0,147^{\mathrm{b}} \pm 0,006$ & 18,33 \\
C & $0,143^{\mathrm{b}} \pm 0,004$ & 17,83 \\
D & $0,126^{\mathrm{b}} \pm 0,005$ & 15,71 \\
E & $0,124^{\mathrm{b}} \pm 0,005$ & 15,46
\end{tabular}


$\mathrm{Na}$ Tabela 3 apresentam-se os resultados encontrados para a estabilidade oxidativa do leite em pó, expressa pelo índice de TBA.

\section{TABELA 3 - ESTABILIDADE OXIDATIVA EXPRESSA PELO ÍNDICE DE TBA}

\begin{tabular}{cc}
\hline Tratamentos & Absorbância a $530 \mathrm{~nm}$ \\
\hline A & $0,120^{\mathrm{a}} \pm 0,015$ \\
B & $0,264^{\mathrm{b}} \pm 0,009$ \\
C & $0,251^{\mathrm{b}} \pm 0,004$ \\
D & $0,189^{\mathrm{c}} \pm 0,013$ \\
E & $0,192^{\mathrm{c}} \pm 0,008$ \\
\hline
\end{tabular}

Médias acompanhadas pela mesma letra não diferem significativamente a p>0,01.

A estabilidade oxidativa do leite em pó, expressa pelo índice de TBA, foi influenciada pela adição das fontes de ferro (Tabela 3), sendo a estabilidade do aminoácido quelato superior à do sulfato ferroso. Não houve diferença significativa $(p>0,01)$ em relação à forma de adição de ferro, antes ou após a secagem do leite, para a mesma fonte de ferro. Esses resultados estão coerentes com os encontrados por MIRANDA (1999) que avaliou o efeito do enriquecimento de ferro em alimento a base de leite em pó e fubá de milho. A oxidação lipídica observada foi maior para o sulfato ferroso que para o ferro aminoácido quelato, com valores médios de absorbância a $532 \mathrm{~nm}$ de 1,356 para o sulfato ferroso e 0,838 para o ferro aminoácido quelato.

Segundo GAUCHERON (2000), sais de ferro produzem grande oxidação de lipídios e os quelatos de ferro geram oxidação menor. Isto pode ser explicado pela menor reatividade do íon ferro na molécula do ferro aminoácido quelato. O íon permanece ligado às duas moléculas de glicina, não estando disponível para catalisar as reações de oxidação lipídica.

A oxidação lipídica causa alterações sensoriais no alimento devido ao desenvolvimento do sabor de ranço e a medida da oxidação lipídica pelo teste de TBA tem sido correlacionada positivamente com a presença de sabores indesejáveis no alimento (GAUCHERON, 2000).

Os resultados observados para a biodisponibilidade de ferro in vitro, medido em $\mathrm{pH} 7,5$, mostraram maior biodisponibilidade do ferro aminoácido quelato $(p<0,01)$ em relação ao sulfato ferroso (Tabela 4). A média da biodisponibilidade in vitro obtida para o ferro aminoácido quelato foi de $31,81 \%$ e para o sulfato ferroso de $17,41 \%$.

TABELA 4 - BIODISPONIBILIDADE DE FERRO IN VITRO E BIODISPONIBILIDADE IN VIVO, DEDUZIDA A PARTIR DA EQUAÇÃO DE RAO E PRABHAVATHI

Tratamentos Biodisponibilidade (\%) in vitro
Biodisponibilidade (\%) in vivo, deduzida

\begin{tabular}{lll}
\hline A & $14,79^{\mathrm{a}} \pm 3,33$ & 7,42 \\
B & $17,76^{\mathrm{a}} \pm 5,54$ & 8,83 \\
$\mathrm{C}$ & $17,07^{\mathrm{a}} \pm 5,87$ & 8,50 \\
$\mathrm{D}$ & $31,91^{\mathrm{b}} \pm 7,72$ & 15,50 \\
E & $31,70^{\mathrm{b}} \pm 7,11$ & 15,40 \\
\hline
\end{tabular}

Médias acompanhadas pela mesma letra não diferem significativamente a p>0,01. 
A média da biodisponibilidade in vivo encontrada pela equação de RAO e PRABHAVATHI (1978) para o ferro aminoácido quelato foi de $15,45 \%$ e para o sulfato ferroso de $8,66 \%$. Não houve diferença significativa $(p>0,01)$ entre a biodisponibilidade in vivo deduzida do sulfato ferroso adicionado ao leite e do ferro naturalmente presente nesse alimento.

A eficácia da suplementação ou fortificação com ferro pode ser predita por estudos de biodisponibilidade (OLIVARES et al., 1997). RAO e PRABHAVATHI (1978), em estudos in vitro, observaram que a presença de ácido ascórbico e extrato de carne aumentam o teor de ferro ionizável em $\mathrm{pH} 7,5$, o qual diminui na presença de fitatos e taninos (semelhante aos efeitos desses fatores na absorção férrea em humanos).

Os resultados obtidos para a absorção do sulfato ferroso mostraram-se superiores aos encontrados por STEKEL et al. (1986). Esses verificaram absorção entre 2,9\% a 5,1\% para o sulfato ferroso em estudos com mono-isótopo e duplo-isótopos em leite bovino, com concentrações variando de $10 \mathrm{mg}$ a $19 \mathrm{mg}$ de ferro por litro. Os resultados encontrados para a biodisponibilidade do ferro aminoácido quelato são consistente com os valores constatados por OLIVARES et al. (1997) que avaliaram a biodisponibilidade desse composto adicionado ao leite e verificaram absorção de $11,1 \%$.

O valor encontrado para a biodisponibilidade do ferro no leite sem fortificação $(7,42 \%)$ foi inferior a 10\%, valor obtido por CARDOSO e PENTEADO (1994). Maior biodisponibilidade do ferro aminoácido quelato também foi encontrada por PINEDA et al. (1994) em comparação com o sulfato ferroso na recuperação de anemia em adolescentes e por CORNBLUTH SZARFAC et al. (2001) em estudo com gestantes.

Com a absorção obtida no presente estudo e assumindo o consumo diário de $750 \mathrm{~mL}$ de leite (OLIVARES et al., 1997) fortificado com $15 \mathrm{mg} / \mathrm{L}$, a quantidade de ferro absorvida diariamente será de 1,74 mg para o ferro aminoácido quelato e de 0,97 mg para o sulfato ferroso. Essa quantidade supera as necessidades diárias de ferro do organismo para crianças, estabelecidas em $1 \mathrm{mg}$ por dia (NRC, 1989). Quantidade menor de ferro poderia ser adicionada ao leite com base nesses valores. Neste caso, o leite seria considerado pela legislação como fonte desse mineral (mínimo 0,75 mg de ferro por $100 \mathrm{~mL}$ de leite reconstituído) (BRASIL, 1998). Menor quantidade de ferro adicionada ao leite diminui os problemas relacionados à oxidação de lipídios e tem sido eficiente na recuperação de crianças anêmicas (OSMAN e AL-OTHAIMEEN, 2002; TORRES, 1996).

\section{CONCLUSÃO}

Os processos tecnológicos de enriquecimento do leite em pó com as duas fontes de ferro utilizadas mostraram-se simples e de fácil execução em laboratório. A forma de enriquecimento, antes ou após a secagem do leite, não interferiu na estabilidade lipídica ou biodisponibilidade do ferro in vitro. A estabilidade oxidativa, ou estabilidade lipídica, do leite enriquecido com ferro aminoácido quelato foi maior que a do sulfato ferroso, porém menor que a do controle. Desta forma, o ferro aminoácido quelato provoca menores alterações oxidativas no alimento, conservando suas características por maior período de tempo. A biodisponibilidade in vitro do ferro aminoácido quelato foi maior que a do sulfato ferroso.

\section{ABSTRACT}

\section{FORMULATION OF IRON-ENRICHED POWDERED MILK}

The objective of this work was to study the enrichment of powdered milk with two different iron sources: ferrous sulfate and aminoacid chelate iron. Iron sources were added before and after milk drying. Reconstitution, oxidative stability and bioavailability of iron sources were studied. Regarding enrichment mode, differences were not found in the studied characteristics. Both technological processes were simple and easy to perform in the laboratory. Oxidative stability of powdered milk was greater when 
aminoacid chelate iron was used and both sources differed from the control. Aminoacid chelate iron showed greater bioavailability in vitro (31.81\%) than ferrous sulfate (17.41\%). The bioavailability deduced in vivo, was $15.45 \%$ for aminoacid chelate iron and $8.66 \%$ for ferrous sulfate. Aminoacid chelate iron revealed better oxidative stability and better bioavailability compared to ferrous sulfate, being a good source to enrich powdered milk.

KEY-WORDS: ANEMIA IRON-DEFICIENCY; NUTRITIONAL AVAILABILITY; CHEMICAL OXIDATION; POWDERED MILK.

\section{REFERÊNCIAS}

1 BRASIL. Agência Nacional de Vigilância Sanitária. Portaria $n^{\circ} 31$ de 13 de janeiro de 1998. Regulamento técnico referente a alimentos adicionados de nutrientes essenciais. Disponível em: http://www.anvisa.gov.br. Acesso em: 08 abr. 2004.

2 BUONGERMINO SOUZA, S.; CORNBLUTH SZARFAC, S.; PACHECO SOUZA, J.M. Anemia no primeiro ano de vida em relação ao aleitamento materno. Revista Saúde Pública, v. 31, n.1, p.15-20, fev. 1997.

3 CARDOSO, M.A.; PENTEADO, M.V.C. Intervenções nutricionais na anemia ferropriva. Caderno de Saúde Pública, v. 10, n.2, p.231-240, abr./jun. 1994.

4 CLAUD, M.V.; FREITAS, O. Compostos alternativos para o tratamento e/ou prevenção da anemia ferropriva. Caderno de Nutrição, v.8, p.1-9, 1994.

5 CORNBLUTH SZARFAC, S.; CASSANA, L.M.N.; FUJIMORI, E.; GUERRA-SHINOHARA, E.M.; OLIVEIRA, M.V. Relative effectiveness of iron bis-glycinate chelate (Ferrochel) and ferrous sulfate in the control of iron deficiency in pregnant women. ALAN - Archivos Latinoamericanos de Nutricion, v.51, n.1, supl.1, p.42-47, Mar.2001.

6 EUCLYDES, R.F. Sistema de análises estatísticas e genéticas - SAEG. Viçosa, MG: UFV. 1983. 68 p.

7 GAUCHERON, F. Iron fortification in dairy industry. Food Science and Technology, v.11, p.403-409, 2000.

8 GOMES, J.C.; SILVA, M.H.L.; SILVA, C.O. Análise de alimentos. Viçosa: Funarbe, 2003. 154 p.

9 IAL. Instituto Adolfo Lutz. Normas analíticas do Instituto Adolfo Lutz. São Paulo, 1985. 533 p.

10 LIMA, A.V.M.S.; LIRA, P.I.C.; ROMANI, S.A.M. et al. Determinant factors of hemoglobin levels in 12 months old infants in the South of the Zona da Mata of Pernambuco. Revista de Saúde Materno Infantil, v. 4, n.1, p.35-43, jan./mar. 2004.

11 MACPHAIL, A.P. Iron deficiency and the developing world. ALAN - Archivos Latinoamericanos de Nutricion, v. 51, n.1, supl.1, p.2-6, Mar.2001.

12 MIRANDA, L.S. Enriquecimento de alimento a base de leite em pó e fubá de milho com ferro. Viçosa, 1999. 73 p. Dissertação (Mestrado em Ciência e Tecnologia de Alimentos), Universidade Federal de Viçosa.

13 NCR. National Research Council. Food and Nutrition Board. Recommended dietary allowances. $10^{\text {th }}$ ed. Washington, 1989. 284 p.

14 OlivARES G. M.; WALTER K.T. Consecuencias de la deficiencia de hierro. Revista Chilena de Nutrición, v.30, n.3, p.226-233, Dic. 2003.

15 OLIVARES, M.; PIZARRO, F.; PINEDA, O.; NAMEM, J.J.; HERTRAMPF, E.; WALTER, T. Milk inhibits and ascorbic acid favors ferrous bis-glycine chelate bioavailability in humans. Journal Nutrition, v.127, p.1407-1411, 1997.

16 OSMAN, A.K.; AL-OTHAIMEEN, A. Experience with ferrous bis-glycine chelate as an iron fortificant in milk. International Journal Nutrition Research, v. 72, n.4, p. 257-263, 2002.

17 PINEDA, O.; ASHMEAD, H. D.; PEREZ, J.M.; LEMUS, C.P. Effectiveness of iron amino acid chelate on the treatment of iron deficiency anemia in adolescentes. Journal of Applied Nutrition, v. 46, n.1/2, p.2-13, 1994.

18 RAO, B.S.N.; PRABHAVATHI, T. An in vitro method for predicting the bioavailabitily of iron from foods. American Journal of Clinical Nutrition, v.31, p.169-175, 1978.

19 SAKAMOTO, L.M. Estudo comparativo entre os aumentos das ferremias, determinados sem administração prévia de ferro; após as administrações de sulfato ferroso e complexo ferro-peptídeo. Ribeirão Preto, 2003. 144 p. Tese (Doutorado em Clínica Médica), Universidade de São Paulo.

20 SOGLIA, S.L.O. Enriquecimento de leite tipo C com ferro aminoácido quelato: biodisponobilidade e características físico-químicas e sensoriais. Viçosa. 1996. 99 p. Dissertação (Mestrado em Ciência e Tecnologia de Alimentos). Universidade Federal de Viçosa. 
21 STEKEL, A.; OLIVARES, M.; PIZARRO, F.; CHADUD, P.; LOPEZ, I., AMAR, M. Absorption of fortification iron from milk formulas in infants. American Journal of Clinical Nutrition, v.43, p.917-922, 1986.

22 TORRES, M.A.A.; LOBO, N.F.; SATO, K.; QUEIROZ, S.S. Fortificação do leite fluido na prevenção e tratamento da anemia carencial ferropriva em crianças menores de 4 anos. Revista de Saúde Pública, v.30, n.4, p.350-357, ago. 1996.

23 TUMA, R.B.; YUYAMA, L.K.O., AGUIAR, J.P.L.; MARQUES, H.O. Impacto da farinha de mandioca fortificada com ferro aminoácido quelato ao nível de hemoglobina de pré-escolares. Revista de Nutrição, v. 16, n.1, p.29-39, jan./mar. 2003. 